\title{
A Case Series of Laparoscopic Duodenojejunostomy for the Treatment of Pediatric Superior Mesenteric Artery Syndrome
}

Bohanon $\mathrm{FJ}^{1}$, Nunez Lopez $\mathrm{O}^{1}$, Graham BM${ }^{1}$, Griffin $\mathrm{LW}^{1}$, Radhakrishnan $\mathrm{RS}^{1,2^{*}}$

${ }^{1}$ Department of Surgery, University of Texas Medical Branch, 301 University Blvd., Galveston, TX, 77555, USA.

${ }^{2}$ Department of Pediatrics, University of Texas Medical Branch, 301 University Blvd., Galveston, TX, 77555, USA.

\section{Abstract}

Superior mesenteric artery syndrome (SMAS) is a rare, debilitating clinical condition caused by compression of the third portion of the duodenum by the superior mesenteric artery. Common symptoms include intermittent postprandial abdominal pain, nausea, weight loss, and bilious vomiting. Here we present a case series of three patients with SMAS who were treated with laparoscopic duodenojejunostomy.

Patients were females between 12-17 years old. All patients underwent a successful laparoscopic duodenojejunostomy after diagnosis. Mean time to feedings after surgery was $4.00 \pm 1.15$ days (mean $\pm \mathrm{SD}$ ) and length of stay was $8.6 \pm 2.7$ days.

SMAS remains a complex disease to diagnose and treat. Here we demonstrate that laparoscopic treatment of SMAS is a safe surgical treatment option, and is associated with earlier initiation of enteral feeds and a shorter hospital stay after surgery when compared to medical treatment. This is a safe, effective, and relatively simple procedure for the experienced minimally invasive surgeon [1].

Keywords: SMA syndrome; Pediatric; Duodenojejunostomy; Weight Loss.

\section{Introduction}

Superior mesenteric artery syndrome (SMAS) is a rare and severely debilitating disease entity caused by an abnormal anatomic relationship between the aorta, mesenteric vessels and the duodenum [2]. The resulting anatomic variation causes compression of the third portion of the duodenum between the aorta and the superior mesenteric artery (SMA). Normally, the duodenum will pass between the SMA and the aorta with the angle between the two vessels ranging from $38-56^{\circ}$. In patients with SMAS the angle is reduced to a range of $6-25^{\circ}$. This reduction in angle is associated with other comorbidities including rapid weight loss, malnutrition, eating disorders, corrective spinal surgery, rapid vertical growth, burn injury, AIDS, cancer and pancreatitis [2-5].

The reported incidence of SMAS varies between 0.013 and $0.3 \%$ [3]. SMAS may present as an acute small bowel obstruction or as chronic intermittent compression of the duodenum. Chronic compression of the duodenum results in a long history of gas- trointestinal symptoms including failure to thrive, nausea, weight loss, early satiety, and bilious vomiting.

The diagnosis of SMAS is obtained by radiographic evidence of vascular obstruction of the duodenum using barium upper gastrointestinal (UGI) series, computed tomography (CT), CT angiography, or magnetic resonance angiography (MRA) [4]. Due to a low index of suspicion, this diagnosis is commonly delayed, resulting in a protracted course of symptoms. One case series reports symptoms lasting from $8-28$ months [2]. Once diagnosed, SMAS can be treated medically or surgically. Medical therapy consists mainly of nutritional therapy, and has a reported success rates of $14-83 \%$ depending upon the study [4]. Currently there are no data suggesting an optimal medical treatment period or indications for surgical therapy.

Surgical options include mobilization of the duodenum by division of the ligament of Trietz (Strong's procedure), duodenojejunostomy with or without division of the fourth part of the

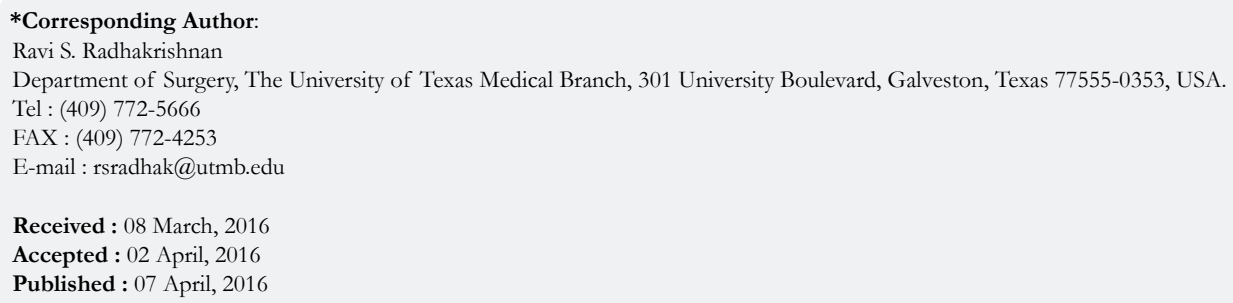

Citation: Ravi S. Radhakrishnan et al.(2016) A Case Series of Laparoscopic Duodenojejunostomy for the Treatment of Pediatric Superior Mesenteric Artery Syndrome. Int J Surg Res. S1:001, 1-5. doi: http://dx.doi.org/10.19070/2379-156X-SI01001

Copyright: Ravi S. Radhakrishnan ${ }^{\circ}$ 2016. This is an open-access article distributed under the terms of the Creative Commons Attribution License, which permits unrestricted use, distribution and reproduction in any medium, provided the original author and source are credited. 
duodenum, or gastrojejunostomy [2]. Strong's procedure is a less invasive, quicker procedure, but can be technically difficult due to adhesions, and caudal displacement of the duodenum is limited due to interference of short vessels from the inferior pancreaticoduodenal artery to the duodenum [6]. Lee and Mangla concluded after reviewing 146 cases operated after 1963 that duodenojejunostomy acheived the best results in severe cases of SMAS and was significantly better when compared to gastrojejunostomy and Strong's procedure [6]. Thus, here we present a case series of three patients with SMAS that were treated with laparoscopic duodenojejunostomy (Table1).

\section{Surgical Technique}

General anesthesia, in combination with regional anesthesia, is employed. The abdominal cavity is entered through the umbilicus, a 5 or $10 \mathrm{~mm}$ port is inserted. After intraperitoneal insufflation, four additional $5 \mathrm{~mm}$ ports are placed under direct vision, two in the right subcostal region and two in the left subcostal region. The transverse colon is mobilized over the liver; a window is created in the mesocolon to allow visualization of the third portion of the duodenum, the ligament of Treitz is identified. A suitable loop of jejunum, 10 to 15 centimeters from the Ligament of Treitz, is approximated to the exposed third portion of duodenum. Longitudinal enterotomies are made in the antimesenteric borders of both duodenum and jejunum. A single layer running anastomosis using absorbable sutures is performed; the anastomosis can also be performed using interrupted sutures. After completion, patency and adequate position of the anastomosis are verified. A nasogastric tube is left in place. The laparoscopic instruments are removed, pneumoperitoneum is evacuated, and the umbilical port fascia site is closed with absorbable sutures. Average procedure time was 2 hours and 22 minutes.

\section{Case 1}

A healthy 12-year-old female presented to the Emergency Department (ED) with a three week history of upper respiratory symptoms. In addition, she complained of anorexia and weight loss. She was diagnosed and treated for Mycoplasma pneumonia. Following successful treatment of her pneumonia, she started developing intermittent abdominal pain. These intermittent episodes of abdominal pain continued to increase in duration and frequency. Seven months following resolution of her upper respiratory tract infection, she presented to her primary care physician for continuing intermittent abdominal pain, nausea, weight loss, and forceful emesis. She was advised to keep a food diary and to eliminate lactose and meat from her diet. Despite changing her diet, she continued to suffer from nausea and projectile vomiting with a five pound weight loss. She was taken to the ED for a diagnostic workup. A CT scan was performed, and was suggestive of SMAS with an acute angle $\left(14^{\circ}\right)$ take off of the SMA causing compression of the left renal vein and the third portion of the duodenum (Figure 1). She was admitted to the hospital for fluid resuscitation and further workup. Intermittent abdominal pain, nausea, and vomiting continued through the duration of her hospital stay. An UGI series was performed, further sug-

Table 1. Case series of three patients with superior mesenteric artery syndrome treated with laparoscopic duodenojejunostomy. Each patient is listed as a case with their age, comorbidities, number of postoperative days before tolerating full feeds, number of postoperative days to discharge and the angle, determined by CT scan, between the superior mesenteric artery and the aorta. Statistics reported as mean \pm standard deviation (SD). SMA: Superior mesenteric artery.

\begin{tabular}{|c|c|c|c|c|}
\hline & Case 1 & Case 2 & Case 3 & Mean \pm SD \\
\hline Age (years) & 12 & 17 & 17 & $15.3 \pm 2.89$ \\
\hline Comorbidities & $\begin{array}{l}\text { Pneumonia,weight loss, } \\
\text { emesis }\end{array}$ & Anorexia nervosa, weight loss & $\begin{array}{l}\text { Tuberous sclerosis,seizures, } \\
\text { scoliosis, failure to thrive }\end{array}$ & \\
\hline Time to Full Feeds (Postoperative Day) & 4 & 3 & 4 & $3.7 \pm 0.56$ \\
\hline Time to Discharge (Postoperative Day) & 5 & 5 & 7 & $5.7 \pm 1.15$ \\
\hline Angle between SMA and Aorta (Degrees) & 14 & 15 & 22 & $17.0 \pm 2.52$ \\
\hline
\end{tabular}

Figure 1. A sagittal image of a computed tomography (CT) of abdomen and pelvis with intravenous contrast. Angle represents that angle between the SMA and the aorta.

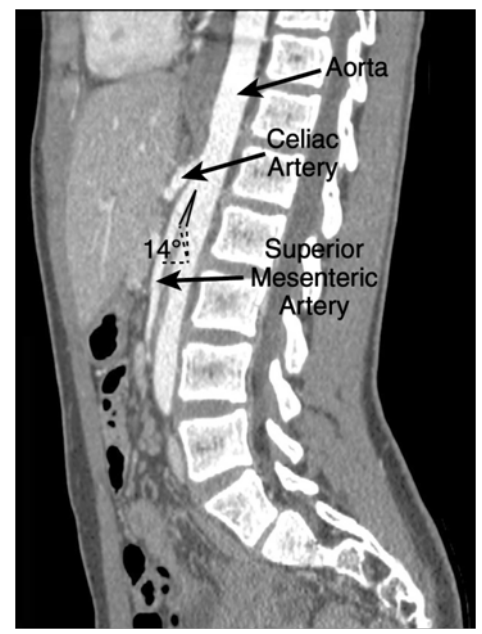


gesting SMAS with a "to-and-fro" peristalsis in the duodenum where the SMA lies. Pediatric surgery was consulted for possible surgical intervention. She was taken to the operating room and a laparoscopic duodenojejunostomy was performed. Her postoperative course was uneventful. She began a clear liquid diet on postoperative day 2 , and a regular diet on postoperative day 4 . On postoperative day 5 she was discharged home. She was seen in clinic at 2 weeks post-surgery with weight gain and no abdominal pain. Since then there have not been any concerns or complications following surgery.

\section{Case 2}

A 17-year-old female presented to our ED for an acute bout of abdominal pain associated with nausea and non-bilious vomiting. Her prior medical history is significant for anorexia nervosa for a period of six years with a body-mass-index (BMI) of 14 at the lowest point. In addition, she was diagnosed with delayed gastric emptying with abdominal pain eight months prior to our encounter. The anorexia was severe with at least one admission for hypotension, tachycardia, and hypothermia requiring three weeks of inpatient treatment. At our encounter, she had a BMI of 17 and was gaining weight. A CT scan performed in the ED revealed a reduced angle $\left(15^{\circ}\right)$ between the SMA and aorta causing compression of the duodenum and left renal vein (Figure 2). The CT scan also revealed mesenteric adenitis. She was treated for the pain and mesenteric adenitis and discharged home. Subsequently, she presented to our outpatient pediatric surgery clinic a few weeks following hospital admission. After discussion with her and her parents, an elective laparoscopic duodenojejunostomy was scheduled due to concerns of the reduced angle between the aorta and SMA. A laparoscopic duodenojejunostomy was performed without complication. The patient began tolerating a clear liquid diet on postoperative day 2 and a regular diet on postoperative day 3 .
She was discharged home, tolerating full meals and having good return of bowel function on postoperative day 5 . She returned to the ED on postoperative day 9 with acute abdominal pain, nausea, and vomiting. A CT scan revealed no abscess or signs of anastomotic leak, but did reveal possible signs of pancreatitis which was confirmed with a lipase level of $2220 \mathrm{U} / \mathrm{L}$. She was admitted and treated for pancreatitis of unknown origin. She was discharged home on hospital day 5 following treatment of the pancreatitis. She is tolerating full feeds, and had resolution of her obstructive symptoms. She is currently gaining weight and having no concerns or complications after the resolution of the SMAS.

\section{Case 3}

A 17-year-old female with a prior medical history of tuberous sclerosis, seizure disorder, and scoliosis presented to our ED one month post corrective surgery for her scoliosis with a two day history of abdominal pain, nausea, decreased PO intake, dehydration, and non-bilious vomiting. A CT scan performed in the ED revealed a dilated stomach and dilated proximal duodenum with an abrupt tapering of the duodenum as it crosses the midline (Figure 3). Also, the findings in the CT images indicated the possibility of pancreatitis. Laboratory values further increased concern for pancreatitis with a lipase level of $4432 \mathrm{U} / \mathrm{L}$ (compared to a value of $2290 \mathrm{U} / \mathrm{L}$ on admission). She was treated for pancreatitis and worsening seizures. A naso-gastric tube was placed for the partial small bowel obstruction along with total-parenteral-nutrition being initiated. On hospital day 10, following resolution of her pancreatitis, a laparoscopic duodenojejunostomy was performed, and gastric feeding tube was placed due to her neurologic status, secondary to tuberous sclerosis. The patient began enteral feeds on postoperative day 4 and was ready for discharge from a surgical perspective on postoperative day 7 . Following surgery, she has been gaining weight and tolerating feeds without any complications.

Figure 2. A sagittal image of a computed tomography (CT) of abdomen and pelvis with intravenous contrast. Angle represents that angle between the SMA and the aorta.

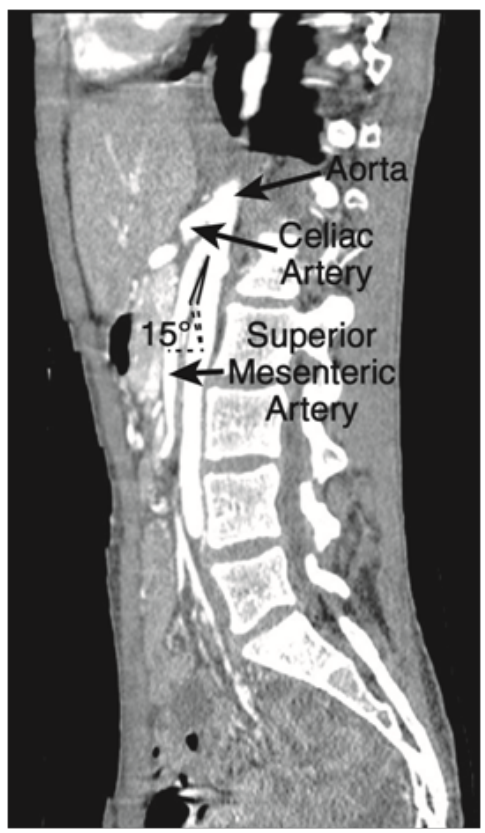


Figure 3. An axial image of a computed tomography (CT) of abdomen and pelvis without intravenous contrast.

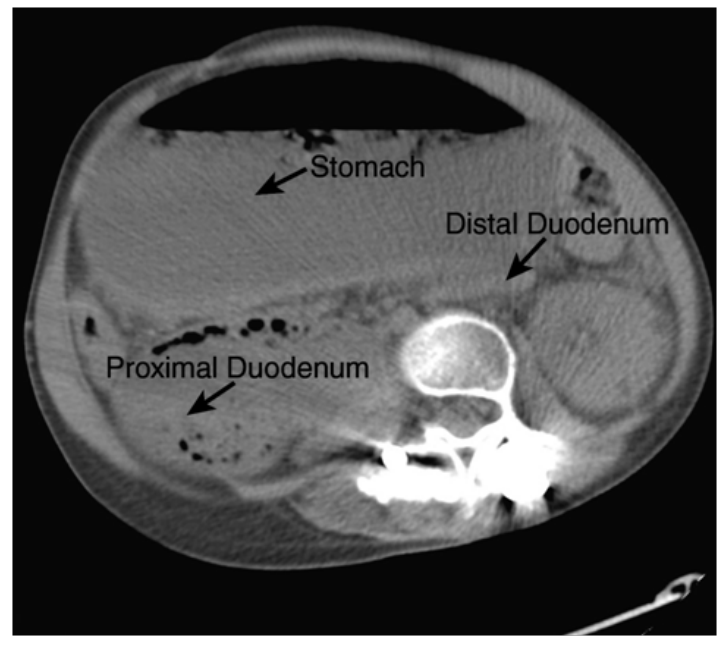

\section{Discussion}

SMAS was first described by Rokitansky in an anatomy text in 1842 [7]. Subsequently, Wilkie published the first comprehensive series of 75 patients in 1927 and his name has become a common eponym for SMAS [6]. It involves the compression of the third portion of the duodenum by an anatomically reduced angle $\left(6-25^{\circ}\right)$ between the SMA and the aorta. Our patients had a mean angle of $14.5 \pm 0.71^{\circ}$. SMAS has been associated with a variety of illness including rapid weight loss, anorexia nervosa, prolonged illness, and corrective spinal surgery (as our three cases demonstrate) $[2-5,8-10]$.

SMAS is more common in females between 10 and 39 years of age and tends to affect younger individuals $[11,12]$. SMAS should be considered when there is a history of rapid weight loss or spinal surgery and when signs and symptoms of upper gastrointestinal obstruction are present. SMAS may present as an acute obstruction requiring urgent therapy or it may have an insidious onset with chronic abdominal pain. Current therapy consists of medical or surgical therapy.

Medical therapy includes postprandial left lateral decubitus positioning, prokinetic medications, anti-reflux medications, fluid resuscitation, parenteral nutrition, and feeding tube placement. Shin et. al. recommends a trial of at least six weeks of medical therapy in children suffering from SMAS before consideration of surgery and the 18 pediatric patients in their retrospective study had a median treatment duration of 45 days [4]. The goal is to increase SMA mesentery and retroperitoneal fat to widen the aortomesenteric angle [11]. While conservative treatment is preferred, there is no clear time limit for the duration of medical treatment, and there have been reports of treatment lasting up to 169 days [6].

Surgical treatment options include mobilization of the duodenum by division of the ligament of Trietz (Strong's procedure), duodenojejunostomy with or without division of the fourth part of the duodenum and gastrojejunostomy. As aforementioned, Strong's procedure may be limited due to adhesions and because caudal displacement of the duodenum is limited due to interference of short vessels from the inferior pancreaticoduodenal artery to the duodenum. Gastrojejunostomy is a feasible treatment, but it does not relieve the proximal duodenal obstruction, and patients can continue to have post-operative obstructive symptoms [11]. A laparoscopic duodenojejunostomy provides a surgical treatment option, without the risks and morbidity of an open surgery.

We demonstrate that laparoscopic duodenojejunostomy may be a safe and effective therapy for SMAS. The shorter hospital stay, low morbidity, and high success of laparoscopically created enteric anastomoses make this approach more conventional and favorable when compared with the traditional open surgery [11]. Our series had one post-operative complication of self-limited pancreatitis of unknown etiology that resolved with medical treatment and observation, while our other patients had no post-operative complications. Our patients had a mean time to eating of $3.7 \pm 0.58$ days and a mean time to discharge of $5.7 \pm 1.15$ days (Table 1). These patients are all doing well post-operatively, tolerating food and gaining weight. Average follow-up for our patients was 16 months.

\section{Conclusion}

SMAS is a severely debilitating disease that is often unrecognized until after a protracted course of symptoms. Current therapy requires long term management, medications, costly parenteral nutrition and rigorous follow-up. We propose that laparoscopic duodenojejunostomy is a safe and effective treatment for SMAS, and should be considered early in the disease course to possibly decrease length of patient suffering and hospital costs. Laparoscopic duodenojejunostomy is a relatively easy operation to perform and may be of benefit because it is a definitive and minimally invasive surgical technique in a duodenal obstruction. [13]

\section{Acknowledgements}

This work was supported by the grant T32-GM008256 (FJB) from the National Institutes of Health. We would also like to thank Karen Martin for her generous help in preparing our data for publication. We have no conflict of interests to disclose.

\section{References}

[1]. Morris TC, Devitt PG, Thompson SK (2009) Laparoscopic duodenojejunostomy for superior mesenteric artery syndrome--how I do it. J Gastrointest Surg 13(10): 1870-1873. 
[2]. Merrett ND, Wilson RB, Cosman P, Biankin AV (2009) Superior mesenteric artery syndrome: diagnosis and treatment strategies. J Gastrointest Surg 13(2): 287-292.

[3]. Bakker ME, van Delft R, Vaessens NA, Rosias PR (2014) Superior mesenteric artery syndrome in a 15 -year-old boy during Ramadan. Eur J Pediatr 173(12): 1619-1621.

[4]. Shin MS, Kim JY (2013) Optimal duration of medical treatment in superior mesenteric artery syndrome in children. J Korean Med Sci 28(8): 12201225 .

[5]. Tsirikos AI, Jeans LA (2005) Superior mesenteric artery syndrome in children and adolescents with spine deformities undergoing corrective surgery. J Spinal Disord Tech 18(3): 263-271.

[6]. Welsch T, Büchler MW, Kienle P (2007) Recalling superior mesenteric artery syndrome. Dig Surg 24(3): 149-156.

[7]. Geer DA (1990) Superior mesenteric artery syndrome. Mil Med 155(7): 321-323.

[8]. Jalilvand A, Fisichella PM (2014) Superior mesenteric artery syndrome. Dig Liver Dis 46(9): 859.

[9]. Mearelli F, Degrassi F, Occhipinti AA, Casarsa C, De Manzini N, et al. (2014) Pinched: superior mesenteric artery syndrome. Am J Med 127(5): 393-394.

[10]. Yakan S, Calıskan C, Kaplan H, Deneclı AG, Coker A (2013) Superior mesenteric artery syndrome: a rare cause of intestinal obstruction. Diagnosis and surgical management. Indian J Surg 75(2): 106-110.
[11]. Pottorf BJ, Husain FA, Hollis HW Jr, Lin E (2014) Laparoscopic management of duodenal obstruction resulting from superior mesenteric artery syndrome. JAMA Surg 149(12): 1319-1322.

[12]. Lee TH, Lee JS, Jo Y, Park KS, Cheon JH, et al. (2012) Superior mesenteric artery syndrome: where do we stand today? J Gastrointest Surg 16(12): 2203-2211.

[13]. Kim IY, Cho NC, Kim DS, Rhoe BS (2003) Laparoscopic duodenojejunostomy for management of superior mesenteric artery syndrome: two cases report and a review of the literature. Yonsei Med J 44(3): 526-529.

Special Issue on

"Neonatal and Pediatric Surgery"

Edited by:

Prakash Kabbur,

Kapiolani Medical Center for Women and Children Honolulu, USA

E-mail: kabburpm@yahoo.com 